\title{
A theoretical model for horizontal convection at high Rayleigh number
}

\author{
G. O. HUGHES ${ }^{1}$, R. W. GRIFFITHS ${ }^{1}$, J. C. MULLARNEY ${ }^{1,2}$ \\ AND W. H. PETERSON† \\ ${ }^{1}$ Research School of Earth Sciences, The Australian National University, Canberra, \\ ACT 0200, Australia \\ ${ }^{2}$ Department of Oceanography, Dalhousie University, Halifax, NS, B3H 4J1, Canada
}

(Received 5 December 2005 and in revised form 27 December 2006)

We present a simple flow model and solution to describe 'horizontal convection' driven by a gradient of temperature or heat flux along one horizontal boundary of a rectangular box. Following laboratory observations of the steady-state convection, the model is based on a localized vertical turbulent plume from a line or point source that is located anywhere within the area of the box and that maintains a stably stratified interior. In contrast to the 'filling box' process, the convective circulation involves vertical diffusion in the interior and a stabilizing buoyancy flux distributed over the horizontal boundary. The stabilizing flux forces the density distribution to reach a steady state. The model predictions compare well with previous laboratory data and numerical solutions. In the case of a point source for the plume (the case which best mimics the localized sinking in the large-scale ocean overturning) the thermal boundary layer is much thicker than that given by the two-dimensional boundary layer scaling of H. T. Rossby (Tellus, vol. 50, 1965, p. 242).

\section{Introduction}

'Filling box' flows (Baines \& Turner 1969) are driven by a localized destabilizing source of buoyancy that maintains a turbulent plume in a confined volume, and have been a useful model of flows in many natural and engineering contexts, for example, in building ventilation (Linden 1999), in magma chambers (Turner 1980) and in the oceans (e.g. Killworth \& Turner 1982). The analysis of Baines \& Turner (1969) shows that the filling box flow reaches an asymptotic state in which the density gradient and velocity fields are statistically steady. Crucially, however, the bulk density of water in the box continues to evolve because the flow is driven by a continuous net supply of buoyancy. The time rate of change of density at any given level is determined by the vertical advection of fluid past that level. However, in many physical applications, where the flow is in a closed volume, this net supply of buoyancy cannot continue unchecked - compensating buoyancy losses will develop and the flow must evolve towards a steady state where the net supply is zero. 'Horizontal convection' constitutes a particularly interesting class of such flows where buoyancy is supplied over a section of a horizontal boundary and removed elsewhere on that same boundary. In a thermally equilibrated flow, the rates of supply and removal are equal. The primary motivation for previous studies of this form of convection (Stommel 1962, Rossby 1965, 1998; 
Beardsley \& Festa 1972; Hignett, Ibbetson \& Killworth 1981; Mullarney, Griffiths \& Hughes 2004) has been to understand the global response of the oceans to net heating and cooling of the sea surface at low and high latitudes, respectively. However, such models may also find application to convective flows in a confined volume in engineering and industrial contexts where the equilibration time scale of the flow is much shorter than the time scale over which the forcing varies.

Differential heating of the horizontal boundary in horizontal convection establishes a thermal boundary layer, which is observed to feed into a plume at one end of the box (Rossby 1965, 1998; Hignett et al. 1981; Mullarney et al. 2004; Wang \& Huang 2005). Otherwise, horizontal convection has much in common with the circulation in the 'filling box' - in both flows, water in the plume penetrates rapidly through the depth of the box to supply a plume outflow along the opposite horizontal boundary, whereupon it becomes part of a broad vertical flow in the box interior towards the level of the plume source. The density anomaly of water in the plume is reduced with vertical distance from the source by entrainment and mixing of water from the box interior into the plume. Consequently, a density stratification is established throughout the depth of the box in both flows. The stratification is strongest at levels near the plume source (the forcing boundary). However, there is an especially large contrast in the case of horizontal convection between the strength of the stratification in the thermal boundary layer and that throughout the remainder of the box interior (Mullarney et al. 2004). Furthermore, the absolute strength of the stratification in the box interior is very weak in high-Rayleigh-number horizontal convection driven by a two-dimensional thermal forcing (Mullarney et al. 2004). Although the interior might be assumed to be unstratified as a first approximation, we discuss in $\S 2$ the dynamical role played by the weak stratification.

In this paper, we develop a new solution for a steady filling box (which we term a 'recycling box') and apply this to describe horizontal convection. We ensure that the density field in the box is in a steady state by applying a stabilizing buoyancy source at the same horizontal boundary as the destabilizing buoyancy source driving the plume. The stabilizing source is of such a strength that the net supply of buoyancy to the box is zero. Unlike the filling box flow, in which vertical diffusion or turbulent mixing are usually neglected, vertical diffusion in the box interior is then important in maintaining the density distribution and in transferring the matching buoyancy fluxes to the fluid.

Rossby (1965), Hignett et al. (1981) and Mullarney et al. (2004) have developed scaling arguments based on their experiments to describe the thermal boundary layer in two-dimensional horizontal convection, involving a buoyancy-viscous momentum balance. Hignett et al. (1981) considered the effect of background rotation and Mullarney et al. (2004) conducted experiments at small aspect ratio and large Rayleigh number. Siggers, Kerswell \& Balmforth (2004) have developed a description of horizontal convection by placing bounds on the heat transport as measured by a Nusselt number. However, in order to predict the temperature distribution and the overturning mass flux (given imposed thermal boundary conditions), it is necessary to construct an explicit solution for the flow.

Manins (1973, 1979), Peterson (1979) and Killworth \& Manins (1980) have developed theoretical models including advection and diffusion in the interior, and which have application to horizontal convection. Manins (1973) considered a turbulent line plume in a rectangular box, a formulation which he extended to describe the overturning circulation in the (approximately wedge-shaped) Red Sea. His predictions for the density profile agreed well with observations, but relied on the adjustment 
of several free parameters. Manins (1979) developed a perturbation analysis for very high Rayleigh number horizontal convection, in which the plume is assumed to be turbulent and to arise from a point buoyancy source. He showed that in the interior, to first order, the isopycnals are horizontal and the vertical motion is independent of horizontal position. He argued, therefore, that detailed modelling of the plume outflow is unnecessary because the outflow rapidly redistributes water from the plume in the horizontal. The solution showed that the variation with depth of the vertical velocity in the interior is almost linear. However, his solutions for the steady-state flow (in which vertical diffusion is important) are valid only reasonably close (within a few thermal boundary-layer thicknesses) to the horizontal surface at which the forcing is applied. Killworth \& Manins (1980) developed a similarity description of horizontal convection driven by a quadratic temperature variation along the horizontal boundary at which the forcing is applied. However, instead of explicitly representing the plume, they extend the result of Manins (1979) and assume that the upwelling velocity is a linear function of depth through the depth of the flow.

Hughes \& Griffiths (2006) have proposed a solution for horizontal convection in the context of the global oceans, where their plumes are dense slope currents on an $f$-plane. Their inviscid solution describes both the plume and the interior throughout the full box depth. Here we modify the theoretical development of Hughes \& Griffiths (2006) by considering a more general dimensionless form based on vertical plumes from line and point sources. This solution is closely related to that developed by Peterson (1979) for a turbulent line plume in a rectangular box.

Most importantly, this approach enables comparison of our model predictions with experimental data for horizontal convection. We also compare our inviscid solution to the results of the viscous boundary-layer analyses for horizontal convection of Rossby (1965) and Mullarney et al. (2004), and show that our theory based on the interior and plume dynamics predicts the same thermal boundary-layer properties and Nusselt number-Rayleigh number relationship. This raises the question of why viscous and inviscid solutions are consistent with each other.

The system of equations that we use to describe the recycling box is developed in $\S 2$, followed in $\S 3$ by the general solutions. The predictions of the model are then compared in $\S 4$ with horizontal convection data from laboratory experiments, and in $\S 5$ we briefly discuss the relevance of buoyancy forcing for the overturning circulation of the oceans. Finally, in $\S 6$ we present our conclusions.

\section{Governing equations}

Without loss of generality, we consider in the remainder of this paper horizontal convection driven by heat fluxes applied at the base of a box, as in the laboratory experiments reported by Rossby (1965), Hignett et al. (1981), Mullarney et al. (2004) and Wang \& Huang (2005). The destabilizing buoyancy source then corresponds to heating and the stabilizing flux to cooling (figures 1 and 2). In each of the experimental studies, the destabilizing and stabilizing fluxes were applied over large areas of the base (e.g. figure 1), but the resulting flow is highly asymmetric with a tightly confined plume at the hottest end of the box. The global overturning circulation in the oceans displays a similar asymmetry (Stommel 1962). These observations suggest a useful approximation: we treat the heating flux at the base (cooling at the ocean surface) as highly localized (as in the filling box model), and allow the cooling at the base (or heating at the ocean surface) to be distributed. The total buoyancy supplied to the plume will be the primary factor determining the vertical structure of the flow, and we 


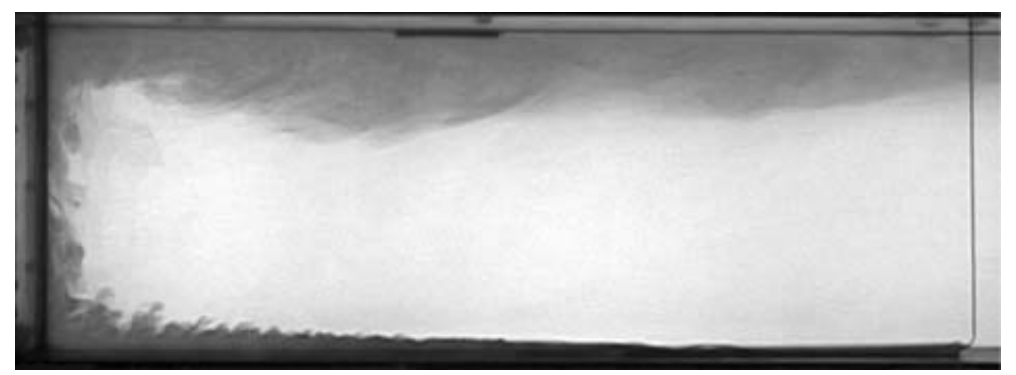

FIgURE 1. Dye visualization of the convective flow driven in a box by differential heating along the base (Mullarney et al. 2004). No net heating of fluid in the box occurs in this experiment - heat is supplied over the left-hand half of the base and removed at the same rate over the right-hand half of the base (see $\S 4$ for details). The field of view shows only the left-hand end of the box. The thin vertical line at the right of the photograph corresponds to the centre of box, where dye was introduced into the boundary layer adjacent to the base. Heat is transferred to/from this boundary layer, maintaining a horizontal density gradient and a flow directed from the cooled region to the heated region (right to left). An eddying outflow from the plume is visible at the top of the box, directed from left to right. Under the large-Rayleigh-number conditions considered in this paper, most of the vertical temperature variation occurs across the bottom boundary layer, which occupies a small fraction of the total depth.

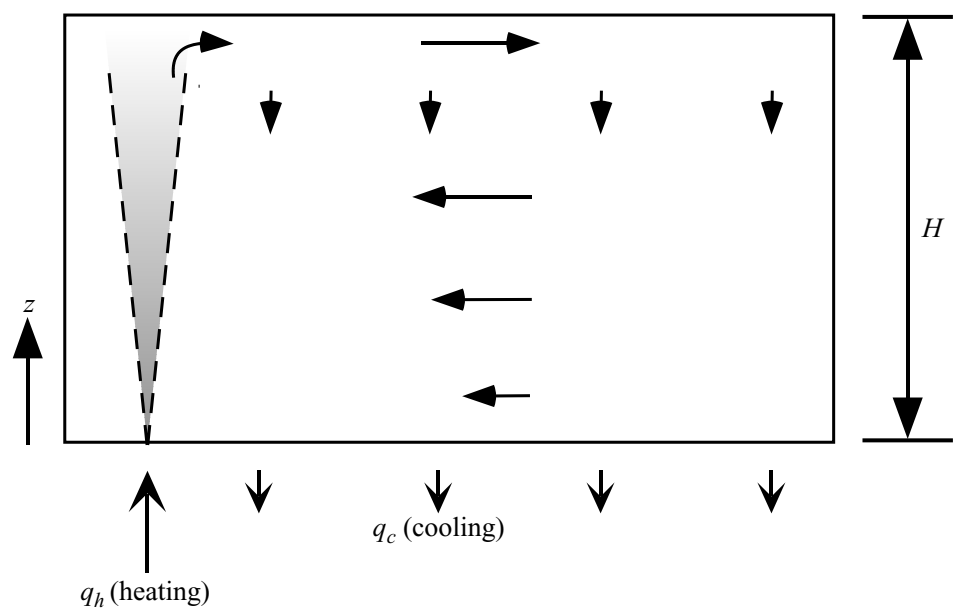

FIGURE 2. Schematic diagram of the 'recycling box' model for horizontal convection. Bottom heating leads to a tightly confined region of upwelling (represented as an isolated vertical plume) from the bottom to the top in a box of depth $H$. Cooling at a rate $q_{c}=-q_{h}$ (per unit spanwise box width for a line plume, or in total for an axisymmetric plume) is distributed uniformly over the base, allowing the flow to reach a steady state. If the rising plume is turbulent, entrainment drives recirculation in the interior.

do not expect either the exact position of the heating or the size of the heated area to play a significant role in this. Thus, provided there is no net heat input, our hypothesis is that the precise nature of the thermal boundary conditions (applied temperature or flux and their distribution along the box) is not important, as the stable thermal boundary layer spreads horizontally to isolate the boundary where thermal forcing is applied from the whole of the interior (which lies above the unstable convective boundary layer seen in figure 1). We return to discuss this approximation in $\S 4$. 
For a line plume (figure 2), the rate of heating $q_{h}$ per unit spanwise box width at the base produces a specific buoyancy flux

$$
F_{h}=\frac{\alpha g q_{h}}{\rho_{r} c_{p}}
$$

per unit spanwise box width, where $\alpha$ is the coefficient of thermal expansion, $g$ is the acceleration due to gravity, $\rho_{r}$ is a reference density and $c_{p}$ is the specific heat capacity. In the case of an axisymmetric plume, the total specific buoyancy flux is also given by (2.1), where $q_{h}$ is instead the total rate of heating. Both the line and axisymmetric plumes are assumed to be turbulent, as in the filling box (Baines \& Turner 1969; Killworth \& Turner 1982; Pierce \& Rhines 1996). Experiments (Mullarney et al. 2004) confirm this to be the case (figure 1) at Rayleigh numbers in excess of $10^{12}$ (based on heat flux and box length).

Although figure 2 depicts an isolated vertical plume in a rectangular box, we will take advantage of symmetry in our model to define an idealized 'half-plume' against the boundary (in order to model the flow in figure 1). (This proves to be a useful model for a line plume against a wall. However, this concept has less direct application for plumes that are not uniform in the spanwise direction because secondary circulations modify the plume geometry, Baines 1985.) The ratio of height to length of the box is assumed to be small so that horizontal velocities are much greater than vertical velocities outside of the plume. We expect in this situation that a one-dimensional (horizontally uniform) model will provide a good description of the flow in the box interior (Manins 1979). Under the one-dimensional approximation, we must distribute the cooling flux uniformly over the base. As horizontal velocities immediately above the base are observed to maintain a thermal and momentum boundary layer of nearly uniform thickness, we also expect the one-dimensional model to describe adequately the vertical structure in the boundary layer. We note, however, that our model neglects the horizontal outflow from the plume, strictly representing only the region of the box below the outflow (as in the filling box model of Baines \& Turner 1969).

The plume is assumed to extend through the full depth of the box, as observed by Rossby (1965, 1998) and Mullarney et al. (2004). There is some current debate, however, as to whether a partial depth circulation can instead be stable (Wang $\&$ Huang 2005). We contend on physical grounds that in a box which has reached thermal equilibrium and is perfectly insulated apart from the single horizontal surface through which heat is exchanged, a full-depth circulation is the only possible stable flow state. This circulation is also consistent with the steady flows found numerically by Paparella \& Young (2002) and Siggers et al. (2004) in high-aspect-ratio (depth to length) boxes at low to moderate Rayleigh number - the full-depth plumes were laminar and there was upwelling everywhere in the interior (for Prandtl numbers greater than one). In an equilibrium state, the warmest fluid in the entire box must be immediately adjacent to the end (closest to the plume) of the heated region of the horizontal surface. In other words, the fluid in the plume must be slightly 'overheated' relative to that in the interior (when there is no net heat input into the box). As the heat extracted over the cooled part of that horizontal surface acts to increase the bulk density of fluid in the remainder of the box (by diffusion of heat down the temperature gradient), the warmest fluid in the box must rise to the top of the box. Alternatively, it can be argued that a stable stratification must exist in the box interior for a partial depth circulation to be maintained. Maintenance of a stable stratification then requires a source of heat to the upper (non-convecting) region of the box. However, this requirement represents a paradox because there is no such heating source - the 
boundaries in the upper region of the box are insulating and heat will be removed from this region by diffusion down the temperature gradient. Consequently, we must conclude that partial depth circulation can be a transient state only, and that the convecting region of the flow will deepen (until it occupies the full depth of the box, e.g. see Jeffreys 1925, and thence supplies heat to the top of the box).

\subsection{Two-dimensional plume model}

We develop our model with a 'half-plume' that rises against the vertical boundary (as is observed in experiments with horizontal convection, Rossby 1965; Mullarney et al. 2004). We define either the box length to be $L(z)$ for a line half-plume or the box cross-sectional area to be $A(z)$ for an axisymmetric half-plume. However, by symmetry the model can also be applied to an isolated plume (figure 2) which does not interact with the confining (vertical) boundaries of the box. For an isolated line plume, the box is defined to have length $2 L(z)$ and $q_{h}$ is the rate of heating per unit spanwise box width. For an isolated axisymmetric plume, the box has cross-sectional area $2 A(z)$ and $q_{h}$ is the total rate of heating. The corresponding model for a turbulent axisymmetric plume is summarized in the Appendix.

Following Baines \& Turner (1969), we assume that profiles of mean velocity $\bar{W}$ and density anomaly $\bar{\rho}-\rho_{e}$ through the plume cross-section are similar at all depths and approximated well by a Gaussian form, i.e.

$$
\begin{gathered}
\bar{W}(x, z)=W_{p}(z) \exp \left[-\frac{x^{2}}{R^{2}(z)}\right], \\
\bar{\rho}(x, z)-\rho_{e}(z)=\left[\rho_{p}(z)-\rho_{e}(z)\right] \exp \left[-\frac{x^{2}}{R^{2}(z)}\right],
\end{gathered}
$$

where $x$ is the distance from the plume axis, $W$ is the vertical velocity and $\bar{\rho}-\rho_{e}$ is the density anomaly in the plume relative to water in the interior at the same height but far removed from the plume. We define the vertical coordinate $z$ such that the plume starts at the origin and $z$ increases in the direction of flow in the plume. We have defined $R / \sqrt{2}$ to be the 1- $\sigma$ lateral plume thickness, and $W_{p}$ and $\rho_{p}-\rho_{e}$ to be the mean vertical velocity and density anomaly on the plume axis, respectively. Upon integrating in the horizontal plane, the equations describing conservation of volume, momentum and buoyancy in the plume can be written (Baines \& Turner 1969)

$$
\begin{gathered}
\frac{\mathrm{d}}{\mathrm{d} z}\left[\sqrt{\pi} R W_{p}\right]=2 U_{e}=2 E W_{p}, \\
\frac{\mathrm{d}}{\mathrm{d} z}\left[\frac{R W_{p}^{2}}{\sqrt{2}}\right]=R \frac{\boldsymbol{g} \cdot \hat{z}\left(\rho_{p}-\rho_{e}\right)}{\rho_{r}},
\end{gathered}
$$

and

$$
\frac{\mathrm{d}}{\mathrm{d} z}\left[\frac{R W_{p} \boldsymbol{g} \cdot \hat{z}\left(\rho_{p}-\rho_{e}\right)}{\sqrt{2} \rho_{r}}\right]=R W_{p} \frac{\mathrm{d}}{\mathrm{d} z}\left[\frac{\boldsymbol{g} \cdot \hat{z}\left(\rho_{r}-\rho_{e}\right)}{\rho_{r}}\right],
$$

where $\boldsymbol{g}$ is the gravitational acceleration vector, $\hat{z}$ is the unit vector in the $z$ direction and $E$ is a constant characterizing the ratio of an entrainment velocity $U_{e}$ to the vertical velocity $W_{p}$ on the plume centreline. The entrainment velocity can be interpreted as the rate of increase with $z$ of the plume volume flux per unit spanwise plume width, and $E$ takes a value of approximately 0.1 for a Gaussian plume (Baines \& Turner 1969; Turner 1973). 


\subsection{Interior model}

We develop a one-dimensional description for the flow in the box interior. Rather than allowing isotherms to outcrop along the horizontal boundary where the thermal forcing is applied, we note that this one-dimensional approximation has the effect of collapsing the cooling/heating (and hence the cross-isotherm flow adjacent to the forcing boundary) to the plume edge.

As in the filling box model of Baines \& Turner (1969), conservation of volume in the box interior is expressed as

$$
\frac{\sqrt{\pi}}{2} R W_{p}=-L(z) W_{e},
$$

where $W_{e}$ is the mean vertical velocity in the interior over the length $L(z)$ of the box. Equation (2.7) holds if the box length is defined to be $2 L$ for isolated plumes and to be $L$ for 'half-plumes' against a vertical boundary in the box.

The density field $\rho_{e}$ in the box interior is allowed to vary with depth (cf. Baines \& Turner 1969). However, in contrast to the filling box flow, $\rho_{e}$ does not vary with time and advection of the density field must instead be balanced everywhere by diffusion of the stratifying property. Thus

$$
L(z) W_{e} \frac{\mathrm{d} \rho_{e}}{\mathrm{~d} z}=\frac{\mathrm{d}}{\mathrm{d} z}\left[L(z) \kappa^{*}(z) \frac{\mathrm{d} \rho_{e}}{\mathrm{~d} z}\right],
$$

where $\kappa^{*}$ is the diffusivity characterizing the vertical transport of the stratifying species. We note that the advection-diffusion balance (2.8), coupled with conservation of volume in the interior (2.7) and buoyancy in the plume (2.6), requires that there is no net buoyancy flux at every level in the box. We also note at this point that we do not know the stability of the flow. Hence, a molecular diffusivity may not be appropriate in the interior. Paparella \& Young (2002) have argued that the flow is non-turbulent in a volume-averaged sense following a rigorous definition of turbulence (in terms of the flow behaviour in the limit $\kappa, v \rightarrow 0$ at a fixed $P r$ ). However, this result does not exclude the possibility of localized vertical mixing at high (but finite) Rayleigh numbers. Our solution will also be valid for the case in which mechanical energy is in some way supplied to small-scale turbulence from an external source (as is believed to be the case in the oceans), with $\kappa^{*}$ in this instance being a turbulent mixing coefficient.

\subsection{Boundary conditions}

The line plume is assumed to be forced purely by a localized (zero width in $x$ ) source of destabilizing buoyancy at $z=0$. The rate at which specific buoyancy is exchanged per unit spanwise box width with the plume at $z=0$ is the specific buoyancy flux $F_{0}$. The volume and momentum fluxes are zero across the boundary where the plume is forced and, under a localized source approximation,

$$
\begin{gathered}
{\left.\left[R W_{p}\right]\right|_{z=0}=0,} \\
{\left.\left[R W_{p}^{2}\right]\right|_{z=0}=0,} \\
R(0)=0 .
\end{gathered}
$$

Therefore,

$$
W_{e}(0)=0
$$


by (2.7). Equations (2.9) and (2.11) show that the vertical velocity in the plume $W_{p}$, and hence the entrainment velocity $U_{e}$ (by (2.4)), must be zero at $z=0$. We ensure that the flow reaches a steady state by supplying stabilizing specific buoyancy to the box either at a rate $F_{c}=F_{0}=F_{h}$ per unit spanwise box width for a half-plume (against a vertical boundary) or at a rate $F_{c}=2 F_{0}=F_{h}$ per unit spanwise box width for an isolated line plume. Here, $F_{h}$ is given by (2.1) and the fluid is assumed to have a linear equation of state. The stabilizing flux could be supplied locally or distributed over some of the horizontal boundary at $z=0$, but we expect the flow to be relatively insensitive to such details because the stabilizing flux results in a stable layer adjacent to the horizontal boundary (Mullarney et al. 2004). Although we describe the flow in terms of the buoyancy (heat) input per unit spanwise width, the actual boundary condition (such as an imposed temperature, a temperature gradient, or an imposed heat flux) is irrelevant, so long as the flow has adjusted to have zero net heat input. As we have developed an interior model that neglects variations in the horizontal, we must distribute the stabilizing flux uniformly over the forcing boundary at $z=0$. Upon integrating the specific buoyancy flux per unit spanwise box width over the plume cross-section and taking the limit as $z \rightarrow 0$, the boundary condition may be written

$$
F_{0}=\left.\frac{\sqrt{\pi}}{2 \sqrt{2}}\left[\frac{R W_{p} \boldsymbol{g} \cdot \hat{z}\left(\rho_{p}-\rho_{e}\right)}{\rho_{r}}\right]\right|_{z=0}=\frac{\boldsymbol{g} \cdot \hat{z} L(0) \kappa^{*}}{\rho_{r}} \frac{\mathrm{d} \rho_{e}(0)}{\mathrm{d} z} .
$$

Equations (2.4)-(2.8) are strictly valid only for the part of the box volume that excludes the plume outflow. Here, we assume that the outflow rapidly redistributes fluid from the plume over the horizontal boundary at $z=H$ (Manins 1979), whereupon the fluid becomes part of the interior. Instead of explicitly modelling the outflow in this formulation, we require that the densities of fluid in the plume and in the interior be equal at $z=H$. Thus, we expect boundary conditions of zero buoyancy flux in each of the plume and the interior at $z=H$, i.e.

$$
F(H)=\left.\frac{\sqrt{\pi}}{2 \sqrt{2}}\left[\frac{R W_{p} \boldsymbol{g} \cdot \hat{z}\left(\rho_{p}-\rho_{e}\right)}{\rho_{r}}\right]\right|_{z=H}=\frac{\boldsymbol{g} \cdot \hat{z} L(H) \kappa^{*}}{\rho_{r}} \frac{\mathrm{d} \rho_{e}(H)}{\mathrm{d} z}=0 .
$$

In practice, this condition cannot be enforced, owing to the neglect of the outflow. However, solutions that satisfy (2.14) to a very good approximation are found over a wide parameter range. We return to discuss this point further in $\S 3$.

\subsection{Solution}

In a similar manner to Baines \& Turner (1969), we define the dimensionless variables $\zeta, r, f_{p}, f_{e}, w_{p}$ and $w_{e}$ in terms of the vertical distance from the plume source, the plume thickness, the plume centreline specific buoyancy, the interior specific buoyancy, the plume centreline velocity and the interior vertical velocity, respectively. In selecting a length scale with which to non-dimensionalize the variables, we assume that the thickness of the thermal boundary layer is much less than the box depth. Under these conditions, we argue that the flow is determined primarily by the boundarylayer thickness, which is set by diffusion. As $\kappa^{*}$ and $L$ appear only as a product in the system of equations (2.4)-(2.8) (if $W_{e}$ is eliminated), dimensional considerations dictate that:

$$
\begin{aligned}
& z=2^{-1 / 6} E^{-1 / 3} F_{0}^{-1 / 6}\left(\kappa^{*} L\right)^{1 / 2} \zeta, \\
& R=2^{5 / 6} E^{2 / 3} \pi^{-1 / 2} F_{0}^{-1 / 6}\left(\kappa^{*} L\right)^{1 / 2} r
\end{aligned}
$$




$$
\begin{gathered}
\frac{\boldsymbol{g} \cdot \hat{z}\left(\rho_{p}-\rho_{e}\right)}{\rho_{r}}=2^{1 / 3} E^{-1 / 3} F_{0}{ }^{5 / 6}\left(\kappa^{*} L\right)^{-1 / 2} f_{p}, \\
\frac{\boldsymbol{g} \cdot \hat{z}\left(\rho_{r}-\rho_{e}\right)}{\rho_{r}}=2^{-1 / 6} E^{-1 / 3} F_{0}{ }^{5 / 6}\left(\kappa^{*} L\right)^{-1 / 2} f_{e}, \\
W_{p}=2^{1 / 3} E^{-1 / 3} F_{0}{ }^{1 / 3} w_{p}, \\
W_{e}=2^{1 / 6} E^{1 / 3} F_{0}{ }^{1 / 6} \kappa^{* 1 / 2} L^{-1 / 2} w_{e} .
\end{gathered}
$$

The sign conventions ensure that the dimensionless quantities $\zeta, r, f_{p}$ and $w_{p}$ are always positive and that $w_{e}$ is always negative (as the vertical velocity in the interior is directed towards decreasing $z$ ). If we set $\rho_{r}=\rho_{e}(0)$, then $f_{e}$ is also always negative. Equation (2.15) (with $\zeta$ set to one) gives a scaling for the thickness $h=2^{-1 / 6} E^{-1 / 3} F_{0}{ }^{-1 / 6}\left(\kappa^{*} L\right)^{1 / 2}$ of the thermal boundary layer, where vertical transports of density by advection and by diffusion are in balance. However, the dimensionless box depth is assumed to be much greater than one (i.e. $\zeta_{H}=H / h \gg 1$ ) for the purposes of the large-Rayleigh-number solution in this paper. Vertical transport of density by advection is then much more important than transport by diffusion in the box interior, as shown by (2.20) and the Péclet number

$$
P e=\frac{W_{e} H}{\kappa^{*}}=\frac{H}{h} w_{e} \gg O(1) .
$$

Note that it may be physically argued that the filling box corresponds to the limit $P e \rightarrow \infty$ (when an advection-diffusion balance is inappropriate anywhere in the flow); however, the boundary condition applied in that case at $z=H$ (instead of (2.14)) is one of non-zero buoyancy flux.

Upon substituting (2.15)-(2.20) into (2.4)-(2.8), we obtain

$$
\begin{gathered}
\frac{\mathrm{d}}{\mathrm{d} \zeta}\left(r w_{p}\right)=w_{p}, \\
\frac{\mathrm{d}}{\mathrm{d} \zeta}\left(r w_{p}^{2}\right)=r f_{p}, \\
\frac{\mathrm{d}}{\mathrm{d} \zeta}\left(r w_{p} f_{p}\right)=r w_{p} \frac{\mathrm{d} f_{e}}{\mathrm{~d} \zeta}, \\
r w_{p}=-\frac{L(\zeta)}{L(0)} w_{e}, \\
\frac{L(\zeta)}{L(0)} w_{e} \frac{\mathrm{d} f_{e}}{\mathrm{~d} \zeta}=\frac{\mathrm{d}}{\mathrm{d} \zeta}\left[\gamma(\zeta) \frac{\mathrm{d} f_{e}}{\mathrm{~d} \zeta}\right]
\end{gathered}
$$

where

$$
\gamma=\frac{\left.\kappa^{*} L\right|_{\zeta}}{\left.\kappa^{*} L\right|_{\zeta=0}} .
$$

Introducing the dimensionless specific buoyancy flux in the plume $\phi(\zeta)=r w_{p} f_{p}$, the boundary conditions in (2.13) and (2.14) reduce to

$$
\begin{gathered}
\phi(0)=\gamma\left[-\frac{\mathrm{d} f_{e}(0)}{\mathrm{d} \zeta}\right]=1, \\
\phi\left(\zeta_{H}\right)=\gamma\left[-\frac{\mathrm{d} f_{e}(1)}{\mathrm{d} \zeta}\right]=0,
\end{gathered}
$$


respectively. Upon using (2.25) to eliminate $w_{e},(2.24)$ and (2.26) can be equated and integrated from $\zeta^{\prime}=0$ to $\zeta^{\prime}=\zeta$ to give

$$
\phi(\zeta)=-\gamma \frac{\mathrm{d} f_{e}}{\mathrm{~d} \zeta}=-\frac{\gamma}{r w_{p}} \frac{\mathrm{d} \phi}{\mathrm{d} \zeta},
$$

where the boundary condition in (2.28) has been applied. Upon writing $f_{p}$ in terms of $\phi$ in (2.23), integrating from $\zeta^{\prime}=0$ to $\zeta^{\prime}=\zeta$, assuming that $\gamma$ is one (i.e. $\kappa^{*} L$ is independent of depth) and applying the boundary conditions in (2.10) and (2.28), we obtain

$$
r^{2} w_{p}^{4}=2(1-\phi),
$$

where

$$
\left.r^{2} w_{p}^{4}\right|_{\zeta_{H}}=2
$$

(The assumption of uniform $\gamma$ is in general required to make analytical progress. However, solutions for variable $\gamma$ may be readily obtained by solving the third-order system given by (2.22), (2.23) and (2.30), for $r, r w_{p}$ and $\phi$.)

We note that (2.30) and (2.31) are also consistent with integrating from $\zeta^{\prime}=\zeta_{H} \rightarrow \infty$ to $\zeta^{\prime}=\zeta$ and applying the boundary condition in (2.29). On physical grounds, we take $r w_{p}^{2}$ to be the positive root of (2.31) with $0 \leqslant \phi \leqslant 1$. Therefore, by (2.22), (2.30) and (2.31), we obtain an ordinary differential equation for the dimensionless plume buoyancy flux $\phi$,

$$
\frac{\mathrm{d}}{\mathrm{d} \zeta}\left[\frac{1}{2}\left(\frac{1}{\phi} \frac{\mathrm{d} \phi}{\mathrm{d} \zeta}\right)^{2}\right]-2^{1 / 2}(1-\phi)^{1 / 2}=0 .
$$

We can integrate (2.33) from $\zeta^{\prime}=0$ to $\zeta^{\prime}=\zeta$ to obtain a purely real first-order o.d.e. for $\phi$,

$$
\frac{\mathrm{d} \phi}{\mathrm{d} \zeta}=-2^{1 / 2} 3^{1 / 3} \phi\left[\tanh ^{-1}\left[(1-\phi)^{1 / 2}\right]-(1-\phi)^{1 / 2}\right]^{1 / 3},
$$

which we integrate numerically. The solutions thus obtained for $\phi$ are expected to become accurate for $\zeta_{H}=H / h \gg 1$. Using (2.25) and (2.30), the solution in the interior for the dimensionless density anomaly and the dimensionless vertical velocity is then given by

$$
\begin{gathered}
w_{e}=\frac{1}{\phi} \frac{\mathrm{d} \phi}{\mathrm{d} \zeta}, \\
\frac{\mathrm{d} f_{e}}{\mathrm{~d} \zeta}=-\phi .
\end{gathered}
$$

The quantities describing the plume are then given by

$$
\begin{gathered}
f_{p}=-\frac{\phi^{2}}{\mathrm{~d} \phi / \mathrm{d} \zeta}, \\
w_{p}=-\frac{2^{1 / 2} \phi(1-\phi)^{1 / 2}}{\mathrm{~d} \phi / \mathrm{d} \zeta} \\
r=\frac{(\mathrm{d} \phi / \mathrm{d} \zeta)^{2}}{2^{1 / 2} \phi^{2}(1-\phi)^{1 / 2}} .
\end{gathered}
$$


In dimensional form, $\phi(\zeta)=F(z) / F_{0}$, so that (2.34) may be written in terms of the specific buoyancy flux,

$$
\frac{\mathrm{d} F(z)}{\mathrm{d} z}=-\frac{2^{2 / 3} 3^{1 / 3} E^{1 / 3} F_{0}{ }^{1 / 6}}{\left(\kappa^{*} L\right)^{1 / 2}} F(z)\left\{\tanh ^{-1}\left[\left(1-\frac{F(z)}{F_{0}}\right)^{1 / 2}\right]-\left(1-\frac{F(z)}{F_{0}}\right)^{1 / 2}\right\}^{1 / 3} .
$$

Similarly, the physical interpretation of the interior and plume properties may be aided by writing (2.35)-(2.39) in dimensional form, thus:

$$
\begin{gathered}
W_{e}=\frac{\kappa^{*}}{F(z)} \frac{\mathrm{d} F(z)}{\mathrm{d} z}, \\
\frac{\mathrm{d}}{\mathrm{d} z}\left[\frac{\boldsymbol{g} \cdot \hat{z}\left(\rho_{r}-\rho_{e}\right)}{\rho_{r}}\right]=-\frac{F(z)}{L \kappa^{*}}, \\
\frac{\boldsymbol{g} \cdot \hat{z}\left(\rho_{p}-\rho_{e}\right)}{\rho_{r}}=-\frac{2^{1 / 2} F^{2}}{L \kappa^{*} \mathrm{~d} F / \mathrm{d} z}, \\
W_{p}=-\frac{2 F\left(F_{0}-F\right)^{1 / 2}}{L^{1 / 2} \kappa^{* 1 / 2} \mathrm{~d} F / \mathrm{d} z}, \\
R=\frac{L^{3 / 2} \kappa^{* 3 / 2}(\mathrm{~d} F / \mathrm{d} z)^{2}}{\pi^{1 / 2} F^{2}\left(F_{0}-F\right)^{1 / 2}} .
\end{gathered}
$$

\section{Results}

The dimensionless solutions for specific buoyancy flux, interior upwelling velocity and interior density anomaly are plotted in figure 3 for a box with a line plume and in figure 4 for a box with an axisymmetric plane. These plots show that the approximate solutions will be valid for $\zeta_{H}=H / h \gtrsim 4$ (when $\phi \rightarrow 0$, satisfying (2.29)). For each of the plume geometries, it is apparent from (2.15) and (A 12) that the boundary-layer thickness $h$ increases (and hence the effective box depth, as measured by $\zeta_{H}$, decreases) with $\kappa^{*}$ and decreases with $F_{0}$. The density gradient in the boundary layer varies accordingly to accommodate the buoyancy flux imposed at $\zeta=0$.

The effect of plume geometry on the solutions can be assessed by comparing figures 3 and 4. For a given Péclet number, which characterizes the vertical transport of density in the interior (recall that the Péclet number $P e=\zeta_{H} w_{e}$ ), it is apparent that the turbulent line plume leads to a thinner boundary layer in the vicinity of $\zeta=0$. This is because entrainment into a turbulent line plume is greater at levels near the plume source, where the line plume has a greater perimeter, relative to an axisymmetric plume, across which to entrain ambient fluid. Consequently, the normalized buoyancy flux $\phi(\zeta)=F(z) / F_{0}$ reduces with $\zeta$ at a lesser rate in an axisymmetric plume.

We summarize the solutions in terms of a number of key quantities (table 1). These are the maximum dimensionless vertical velocity $w_{e}\left(\zeta_{H}\right)$ and dimensionless top-tobottom density difference $\Delta f_{e}\left(\zeta_{H}\right)$ in the interior, the dimensionless thickness $\delta$ of the thermal boundary layer that incorporates $95 \%$ and $1-e^{-1}$ (denoted by subscripts 0.95 and $e$, respectively) of the overall top-to-bottom density difference, and the dimensionless vertical velocities $w_{e}$ in the boundary layer at the dimensionless heights $\delta_{0.95}$ and $\delta_{e}$. The asymptotic dependence of dimensionless vertical velocity $w_{e}$ upon $\zeta$ may be predicted by noting that for $\zeta \gtrsim 4$, the dimensionless specific buoyancy flux becomes very small. Thus, (2.22) or (A 18), (2.35) and (2.38) can be used to 

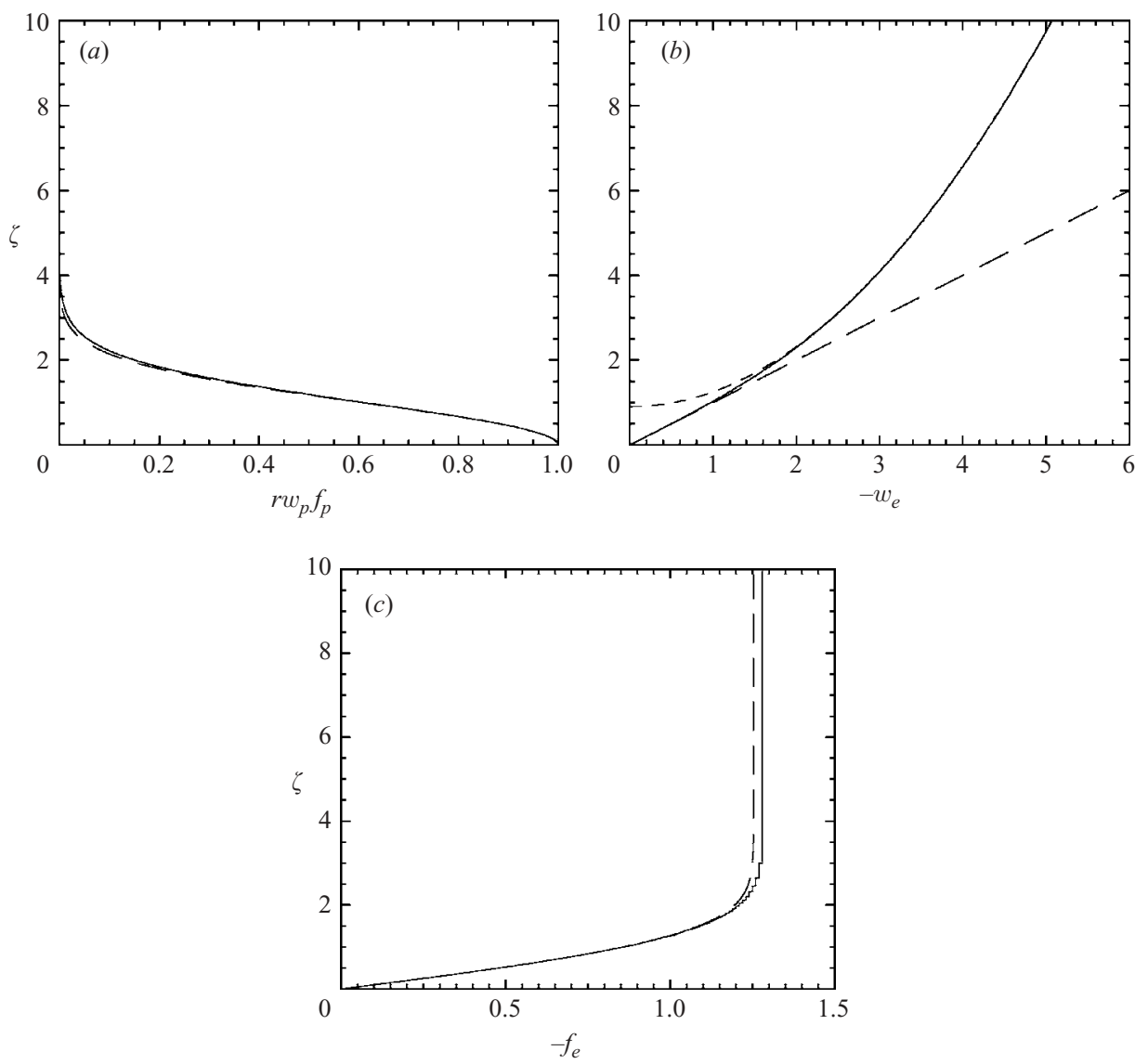

FIGURE 3. Solutions for the recycling box flow driven by a two-dimensional plume: $(a)$ dimensionless specific buoyancy flux, $(b)$ dimensionless interior upwelling velocity, and $(c)$ dimensionless interior density anomaly (relative to the level of the plume source). The long dashed lines show the predicted asymptotic dependence of the solution for $1-\phi \ll 1$ (small $\zeta)$ and the short dashed line in $(b)$ shows the fit of the predicted asymptotic dependence of dimensionless interior upwelling velocity for $\phi \rightarrow 0$ (large $\zeta$ ).

show that

$$
\begin{gathered}
\frac{\mathrm{d}}{\mathrm{d} \zeta}\left(w_{e}^{2}\right) \rightarrow 2^{3 / 2}, \\
\frac{\mathrm{d}}{\mathrm{d} \zeta}\left(w_{e}\right) \rightarrow-2^{1 / 4},
\end{gathered}
$$

for a line plume and an axisymmetric plume, respectively. As is apparent from the asymptotic fits in figures $3(b)$ and $4(b)$, the plume evolution approaches that of an entraining turbulent jet with an apparent momentum source at $\zeta \approx 1$. The dependence of the maximum dimensionless vertical velocity upon $\zeta_{H}$ (in contrast to the dimensionless vertical velocity in the boundary layer, which is independent of $\zeta_{H}$ ) reflects the role of entrainment into the plume in the recycling box flow. As we expect the maximum overturning streamfunction to scale with $w_{e}\left(\zeta_{H}\right)$, the results in table 1 suggest that the flux recycled through the plume in horizontal convection will be larger than the flux in the boundary layer adjacent to the thermally forced surface by an approximate factor that scales with $\zeta_{H}^{1 / 2}$ in the case of a line plume, and with 

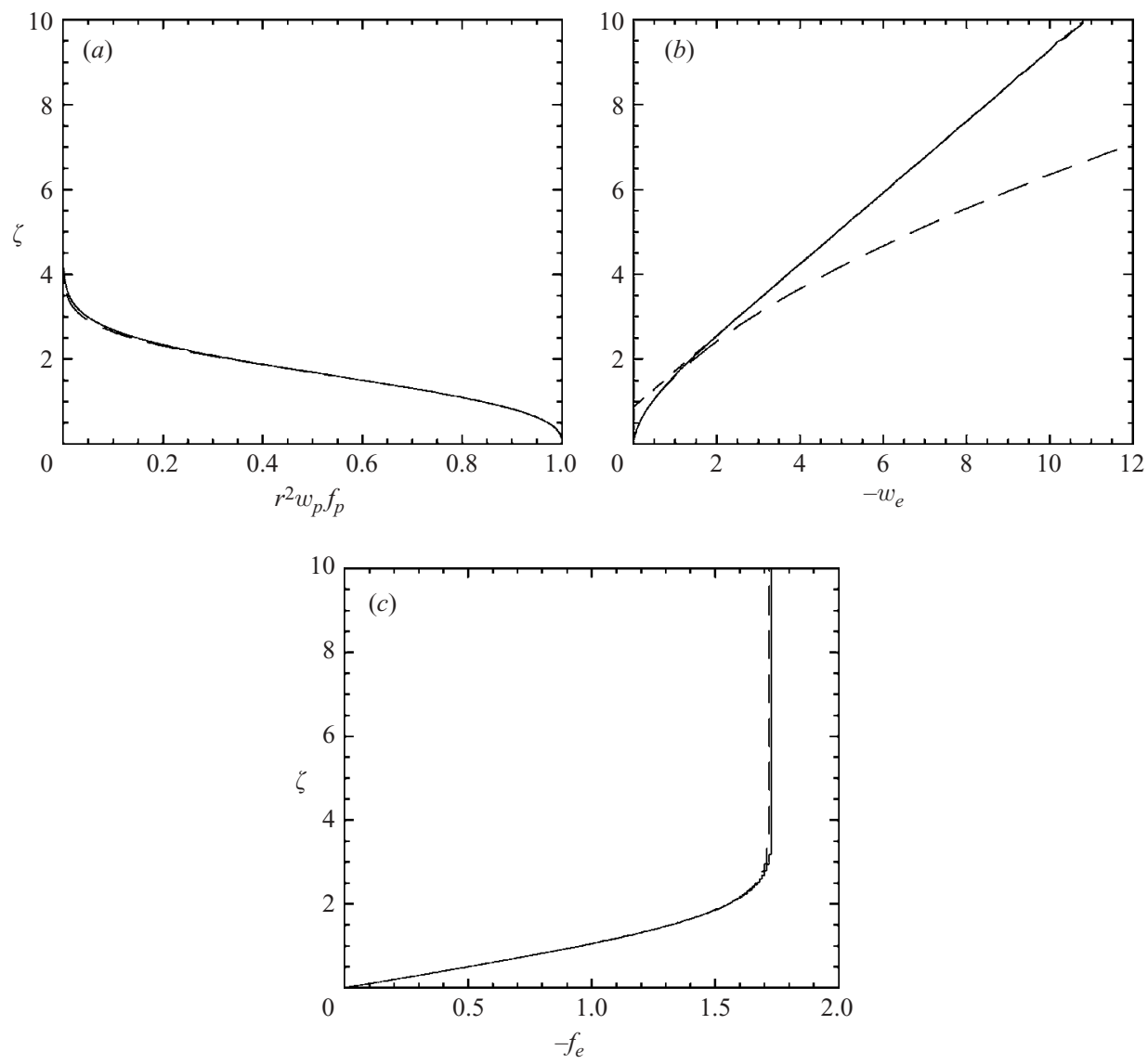

FIGURE 4. As for figure 3, but driven by an axisymmetric plume.

$\begin{array}{rrrr} & \text { Line plume } & \text { Axisymmetric plume } \\ w_{e}\left(\zeta_{H}\right)= & 2^{3 / 4}\left(\zeta_{H}-0.903\right)^{1 / 2} & 2^{1 / 4}\left(\zeta_{H}-0.874\right) \\ \Delta f_{e}\left(\zeta_{H}\right) & 1.28 & 1.73 \\ \delta_{0.95} & = & 2.06 & 2.36 \\ \delta_{e} & = & 0.93 & 1.17 \\ w_{e}\left(\delta_{0.95}\right)= & 1.83 & 1.79 \\ w_{e}\left(\delta_{e}\right)= & 0.90 & 0.60\end{array}$

TABLE 1. Summary of recycling box model solutions for turbulent line and axisymmetric plumes (valid for $\zeta_{H}=H / h \gtrsim 4$ ).

$\zeta_{H}$ in the case of an axisymmetric plume. Although the dimensionless thicknesses $\delta_{0.95}$ and $\delta_{e}$ of the boundary layer have been defined as that region which incorporates a substantial fraction $\left(0.95\right.$ or $1-e^{-1}$, respectively) of the top-to-bottom density difference, we find that the specific buoyancy flux also takes well-defined values at these levels. In particular, $\phi\left(\delta_{0.95}\right)=F\left(h_{0.95}\right) / F_{0} \approx 0.14$ and $\phi\left(\delta_{e}\right)=F\left(h_{e}\right) / F_{0} \approx 0.65$.

Asymptotic approximations can also be found for the solution in the thermal boundary layer. In the case of a line plume, (2.34) can be rearranged using (2.35), and 
expanded for small $(1-\phi)$ to give $w_{e}=-2^{1 / 2}(1-\phi)^{1 / 2}[1+O(1-\phi)]$, which upon substitution into (2.33) yields

$$
w_{e} \approx-\zeta, \quad(1-\phi) \ll 1
$$

Together with (2.35) and (2.36), (3.3) gives

$$
\begin{gathered}
\phi \approx \exp \left(-\frac{\zeta^{2}}{2}\right), \quad(1-\phi) \ll 1, \\
f_{e} \approx-\sqrt{\frac{\pi}{2}} \operatorname{erf}\left(\frac{\zeta}{\sqrt{2}}\right), \quad(1-\phi) \ll 1 .
\end{gathered}
$$

The corresponding results for an axisymmetric plume are

$$
\begin{gathered}
w_{e} \approx-\frac{3^{5 / 3}}{2^{2 / 3} 5^{4 / 3}} \zeta^{5 / 3}, \quad(1-\phi) \ll 1, \\
\phi \approx \exp \left(-\frac{3^{8 / 3}}{2^{11 / 3} 5^{4 / 3}} \zeta^{8 / 3}\right), \quad(1-\phi) \ll 1, \\
f_{e} \approx-\int_{0}^{\zeta} \exp \left(-\frac{3^{8 / 3}}{2^{11 / 3} 5^{4 / 3}} \zeta^{8 / 3}\right) \mathrm{d} \zeta, \quad(1-\phi) \ll 1 .
\end{gathered}
$$

These asymptotic expressions for small $(1-\phi)$ are plotted in figures 3 and 4 and are good approximations for the dimensionless specific buoyancy flux and the dimensionless interior density anomaly over a wide range of $\zeta$.

The Péclet number $P e=W_{e} H / \kappa^{*}=\zeta_{H} w_{e}$ can be used to estimate the ventilation time $\tau$ of the recycling box with a line plume as

$$
\frac{\tau \kappa^{*}}{H^{2}} \sim P e^{-1} \sim\left(\zeta_{H} w_{e}\right)^{-1} \sim \zeta_{H}^{-3 / 2},
$$

and with an axisymmetric plume as

$$
\frac{\tau \kappa^{*}}{H^{2}} \sim P e^{-1} \sim\left(\zeta_{H} w_{e}\right)^{-1} \sim \zeta_{H}^{-2},
$$

assuming that $\zeta_{H} \gg 1$ and using the asymptotic dependences for $w_{e}\left(\zeta_{H}\right)$ from table 1 . Thus, (3.9) and (3.10) show that for values of $\zeta_{H} \gg 1$ (typical of horizontal convection laboratory experiments), the recycling box will be ventilated on a time scale much shorter than that characterizing molecular diffusion through the depth (i.e. $H^{2} / \kappa^{*}$ ).

\section{Recycling box model predictions and horizontal convection}

We now assess whether the recycling box is a useful model to describe horizontal convection. Both the model solution for a specific case and the predicted dependence of the recycling box upon the governing parameters are compared with horizontal convection data from the laboratory experiments and numerical solutions of Mullarney et al. (2004).

The experimental tank and computational domain of Mullarney et al. (2004) were $1.25 \mathrm{~m}$ long, $0.15 \mathrm{~m}$ wide and $0.2 \mathrm{~m}$ high. In one of the cases of horizontal convection that they examined, a heating power per unit spanwise tank width $q_{h}=933 \mathrm{~W} \mathrm{~m}^{-1}$ was supplied through the left-hand half of the tank base, where a constant and uniform heat flux was imposed. A constant and uniform temperature $\left(16^{\circ} \mathrm{C}\right)$ was applied over the right-hand half of the base. By (2.1), the heat input gives a rate of generation of specific buoyancy per unit spanwise tank width of $F_{h}=F_{0}=7.1 \times 10^{-7} \mathrm{~m}^{3} \mathrm{~s}^{-3}$, where 
we have taken $g=9.8 \mathrm{~m} \mathrm{~s}^{-2}$ and water properties at the average interior temperature of $32^{\circ} \mathrm{C}$ as given by Mullarney et al. (2004) (see their table 1 : the specific heat $c_{p}=4177.6$ $\mathrm{J} \mathrm{kg}^{-1} \mathrm{~K}^{-1}$, the coefficient of thermal expansion $\alpha=3.2394 \times 10^{-4}{ }^{\circ} \mathrm{C}^{-1}$, the vertical diffusivity $\kappa^{*} \equiv \kappa_{T}=1.474 \times 10^{-7} \mathrm{~m}^{2} \mathrm{~s}^{-1}$ and $\rho_{r}=994.94 \mathrm{~kg} \mathrm{~m}^{-3}$ ). We note, however, that the equation of state is nonlinear and that at the cooling boundary temperature (approximately the lowest temperature in the tank), the expansion coefficient is approximately half that at $32^{\circ} \mathrm{C}$. We choose the value relevant to the dynamics of the plume and the interior, while recognizing that it is unclear how a different expansion coefficient in the cold boundary layer might influence the flow. The numerical solutions for horizontal convection of Mullarney et al. (2004) with both constant $\alpha$ and the actual equation of state for water show no significant dependence upon $\alpha$. We examine the sensitivity of the predicted tank profiles to expansion coefficient below.

We present in figure 5 the theoretical predictions for the normalized buoyancy flux per unit spanwise box width and the lateral thickness of the plume and for the vertical velocity and temperature profile in the box interior, since these are the quantities of most practical interest. The solutions have been obtained using an entrainment coefficient $E=0.1$ and a coefficient of thermal expansion $\alpha=3.24 \times$ $10^{-4}{ }^{\circ} \mathrm{C}^{-1}$. An additional solution is obtained for $\alpha=1.5 \times 10^{-4}{ }^{\circ} \mathrm{C}^{-1}$, which is the expansion coefficient at $15^{\circ} \mathrm{C}$. The vertical velocity in the box interior increases with height owing to entrainment into the plume (equation (2.7)). The buoyancy flux reduces very rapidly with height owing to entrainment as the plume passes through the strong density stratification in the thermal boundary layer above the bottom of the box. The boundary-layer thickness $h_{0.95}$ under these conditions is predicted to be less than one-tenth of the box depth, i.e. $h_{0.95}=18 \mathrm{~mm}$, such that the dimensionless box depth $\zeta_{H}=H / h \approx 22.9$. Immediately above the boundary layer, the buoyancy flux is small and continues to decrease with height. Thus, it is apparent that the boundary condition at $z=H$ (equation (2.14)) is indeed approximately satisfied by this solution. The variation with height of the buoyancy flux is in stark contrast to the filling box flow, where $F / F_{0}$ would reduce linearly from a value of one at $z=0$ to zero at $z=H$.

As the vertical velocities in the laboratory convection tank are very small, only temperatures are readily measurable. The variation of temperature with horizontal position in the tank is generally weak, except in the boundary layer above the heated and cooled regions of the base. Therefore, we compare the temperature profile predicted by the recycling box model (which ignores horizontal variations in the box interior) with a measured profile for horizontal convection above the cooled base, where the boundary flux is stabilizing. In addition to the measured profile plotted in figure $5(d)$, the horizontally averaged temperature profile from the numerical simulations of Mullarney et al. (2004) is also shown. Both the thermal boundary-layer thickness and the top-to-bottom temperature difference are predicted well. Although it is not visible on the linear scale in figure $5(d)$, all profiles show that the box interior (above the boundary layer) supports a stable temperature stratification. Despite the temperature gradient in the interior being many times smaller than in the boundary layer, it is dynamically significant since it implies that the plume must penetrate to the top of the box to maintain that gradient.

The normalized buoyancy flux per unit spanwise box width in the plume calculated from the previous numerical simulations for horizontal convection of Mullarney et al. (2004) is compared in figure 5(a) with the recycling box prediction for the above experimental parameters. Above the region in the thermal boundary layer, where the flow structure differs from the point-source plume in the recycling box model, the prediction correctly describes over more than two orders of magnitude the decrease 

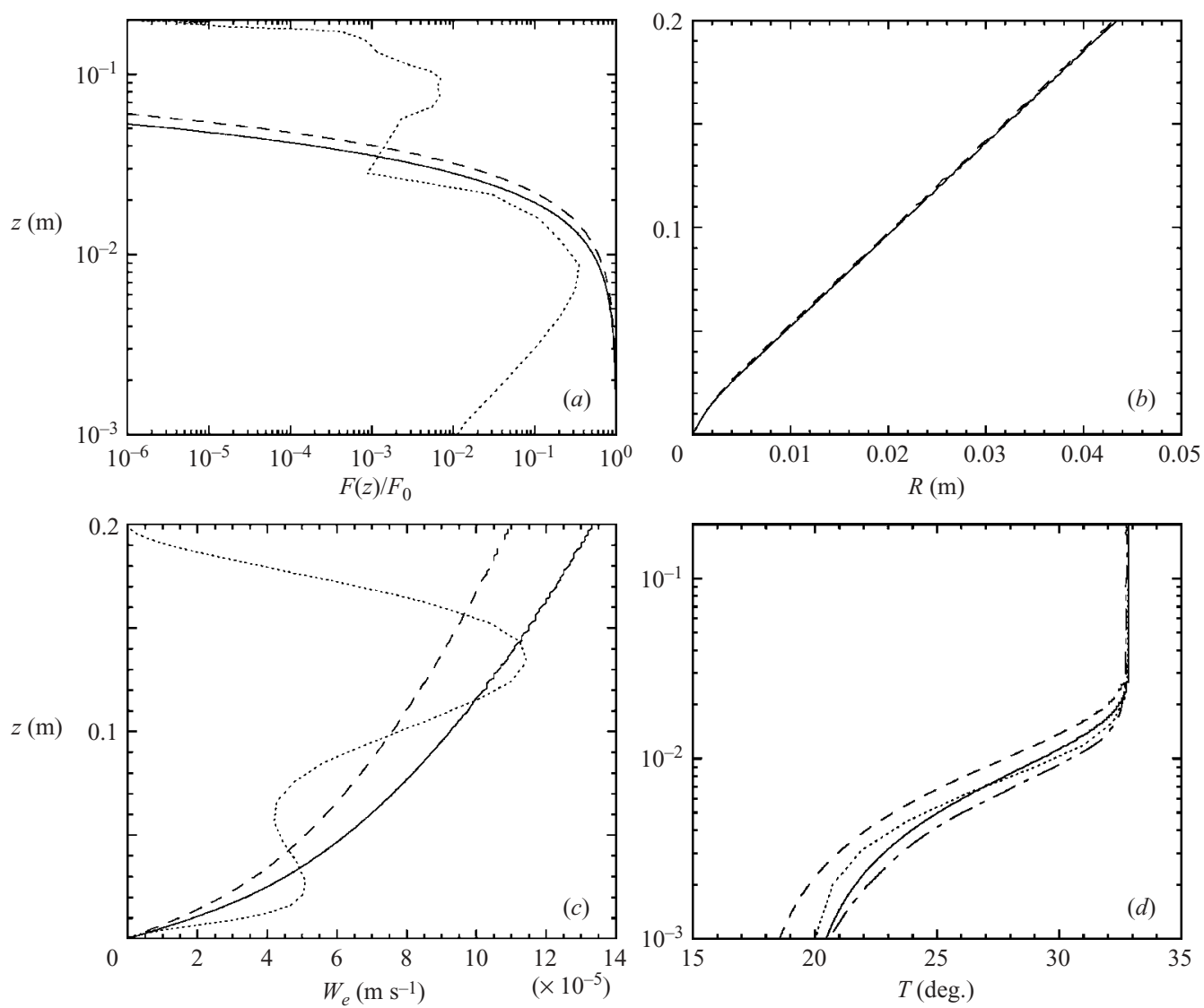

FIGURE 5. Recycling box predictions for $\alpha=1.5 \times 10^{-4}{ }^{\circ} \mathrm{C}^{-1}(---)$ and $\alpha=3.24 \times 10^{-4}{ }^{\circ} \mathrm{C}^{-1}$ (-), appropriate to the horizontal convection experiments of Mullarney et al. (2004): (a) buoyancy flux per unit spanwise width normalized by that supplied by the heating at the base, $(b)$ lateral thickness of the two-dimensional plume, $(c)$ interior vertical velocity, and $(d)$ interior temperature profile. Also plotted on $(a)$ and $(c)$ are the normalized plume buoyancy flux per unit spanwise width and the upwelling velocity profile, respectively, from the numerical

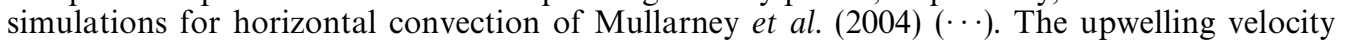
profile is obtained by averaging $W_{e}(x, z)$ in the horizontal between $x=0.16 \mathrm{~m}$ (outside the plume) and $x=1.24 \mathrm{~m}$ (close to the box endwall furthest from the plume). Also plotted on $(d)$ are temperature profiles from the horizontal convection experiments $(--)$ and numerical simulations $(\cdots)$ of Mullarney et al. (2004) obtained above the cooled portion of the tank base. The temperature profile from the numerics is obtained by averaging $T(x, z)$ in the horizontal between $x=0.16 \mathrm{~m}$ and $x=1.24 \mathrm{~m}$. The measured temperatures well above the base are used as a reference for the theoretical prediction and the numerical simulation.

with height of buoyancy flux in the plume. The predicted vertical velocity profile for the recycling box in figure $5(c)$ is consistent with the previous numerical simulations of horizontal convection (Mullarney et al. 2004, figure 10b) for the above experimental parameters. Although the simulations indicate a complicated flow structure, the horizontally averaged downwelling below the level of the outflow tends to increase with height as a consequence of entrainment into the plume. Both the recycling box predictions and horizontal convection simulations in figure 5(c) suggest maximum vertical velocities of approximately $0.13 \mathrm{~mm} \mathrm{~s}^{-1}$. On this basis, we would estimate the ventilation time scale $\tau \sim 2 H / W_{e}(\max ) \approx 50 \mathrm{~min}$ to characterize the recycling of the 
box volume through the plume. This time scale is consistent with observations of the time required for the dye in figure 1 to be spread throughout the laboratory convection tank. In contrast, a ventilation time scale $2 \mathrm{H} / \mathrm{W}_{e}\left(h_{0.95}\right)$, based on the volume flux into the thermal boundary layer (as also predicted by the Rossby (1965) scaling analysis for horizontal convection), is approximately $210 \mathrm{~min}$. For comparison, the time scale for diffusion through the box depth is $H^{2} / \kappa^{*} \sim 75 \mathrm{~h}$.

In order to indicate the sensitivity of horizontal convection to the spatial distribution of heating and cooling at the base, we have carried out an additional numerical simulation for the same parameters as used by Mullarney et al. (2004), as described above. However, the heating power per unit spanwise width $q_{h}=933 \mathrm{~W} \mathrm{~m}^{-1}$ was instead supplied over just $0.15 \mathrm{~m}$ of the $1.25 \mathrm{~m}$ base and the cooling was applied over $1.05 \mathrm{~m}$ of the base (with a $0.05 \mathrm{~m}$ thermal spacer between the regions of heating and cooling). The numerical results suggest that the plume behaviour is mildly sensitive to the change in boundary conditions, but the flow outside of the plume remains largely unchanged. Indeed, the horizontally averaged temperature profiles would coincide in figure $5(d)$ (hence the profile from only the original simulation is plotted). The profile of normalized buoyancy flux in figure $5(a)$ is also virtually unchanged, lending support to the notion that it is the total buoyancy supplied to the plume that is of most importance in setting the vertical structure of the flow. It is on this basis that we expect that the point-source approximation in the recycling box will provide a reasonable model for horizontal convection.

We now express the predictions of the recycling box model in terms of the Rayleigh number and compare the results with the boundary-layer scalings for horizontal convection found by Rossby (1965) (in terms of boundary temperature) and Mullarney et al. (2004) (in terms of the heat flux) and with the laboratory measurements and numerical solutions for horizontal convection of Mullarney et al. (2004). Mullarney et al. (2004) defined a flux Rayleigh number $R a_{F}$ based on the tank length and the heat flux $F_{T}$ per unit area (applied over $0.6 \mathrm{~m}$ of the $1.25 \mathrm{~m}$ base):

$$
R a_{F}=\frac{g \alpha F_{T} L^{4}}{\rho_{r} c_{p} \kappa^{* 2} v} .
$$

Their experiments covered the range $6.5 \times 10^{12} \leqslant R a_{F} \leqslant 6.8 \times 10^{14}$. Using the notation in this paper, $R a_{F}=F_{0} L^{3} / c v \kappa^{* 2}$ and $q_{h}=c F_{T} L$ with $c=0.6 / 1.25$. Thus, using the results in table 1 together with (2.15), (2.18) and (2.20) gives

$$
\begin{gathered}
\frac{h_{0.95}}{L}=2.06 \times 2^{-1 / 6} E^{-1 / 3} c^{-1 / 6} \operatorname{Pr}^{-1 / 6} \operatorname{Ra}_{F}{ }^{-1 / 6}, \\
\left.\frac{\rho_{r}-\rho_{e}}{\rho_{r}}\right|_{\max }=\left.\alpha \Delta T\right|_{\max }=1.28 \times 2^{-1 / 6} E^{-1 / 3}\left(\frac{\kappa^{* 2}}{g L^{3}}\right) c^{5 / 6} \operatorname{Pr}^{5 / 6} \operatorname{Ra}_{F}^{5 / 6}, \\
\frac{W_{e}(H) L}{\kappa^{*}}\left(\equiv \frac{\left.\psi\right|_{\max }}{\kappa^{*}}\right) \approx 2 E^{1 / 2} c^{1 / 4}\left(\frac{H}{L}\right)^{1 / 2} \operatorname{Pr}^{1 / 4} \operatorname{Ra}_{F}^{1 / 4}, \quad \zeta_{H} \gg 1,
\end{gathered}
$$

respectively, where $\left.\psi\right|_{\max }$ is the streamfunction maximum. These results for the recycling box are summarized in table 2. Substituting the values $E=0.1$ and $\operatorname{Pr}=5.17$, we find that (4.2) predicts $h_{0.95} / L=3.39 R a_{F}{ }^{-1 / 6}$, which agrees well with the $h_{0.95} / L=2.65 R a_{F}^{-1 / 6}$ dependence found for horizontal convection in the laboratory experiments by Mullarney et al. (2004) and also with $h_{0.95} / L=2.87 R a_{F}^{-1 / 6}$ given by their numerical solutions. The Péclet number (4.4) based on the vertical velocity $W_{e}(H)$ appears at first glance to be inconsistent with the $R a_{F}^{1 / 6}$ dependence for vertical 


\begin{tabular}{|c|c|c|}
\hline & $R a_{F}$ & $E, q_{h}, \kappa^{*}$ \\
\hline$\left.W_{e}(H) \sim \psi\right|_{\max } \sim$ & $R a_{F}^{1 / 4}$ & $E^{1 / 2} q_{h}{ }^{1 / 4} \kappa^{* 1 / 4}$ \\
\hline$\frac{\rho_{r}-\rho_{e}}{\rho_{r}} \mid \sim$ & $R a_{F}^{5 / 6}$ & $E^{-1 / 3} q_{h}^{5 / 6} \kappa^{*-1 / 2}$ \\
\hline$\left.\rho_{r}\right|_{\max } ^{h_{0.95, e}} \sim$ & $R a_{F}^{-1 / 6}$ & $E^{-1 / 3} q_{h}^{-1 / 6} \kappa^{* 1 / 2}$ \\
\hline$W_{e}\left(h_{0.95, e}\right) \sim$ & $R a_{F}^{1 / 6}$ & $E^{1 / 3} q_{h}^{1 / 6} \kappa^{* 1 / 2}$ \\
\hline$U\left(h_{0.95}\right) \sim$ & $R a_{F}^{1 / 3}$ & $E^{2 / 3} q_{h}^{1 / 3}$ \\
\hline$N u \sim$ & $R a_{F}^{1 / 6}$ & $E^{1 / 3} q_{h}{ }^{1 / 6} \kappa^{*-1 / 2}$ \\
\hline
\end{tabular}

TABLE 2. Summary of power-law dependences of the solutions for a turbulent line plume upon Rayleigh number $R a_{F}$ and upon the entrainment coefficient, heat input per unit spanwise width and interior diffusivity $E, q_{h}$ and $\kappa^{*}$, respectively.

\begin{tabular}{ccccc} 
& & \multicolumn{3}{c}{ Constants } \\
\cline { 3 - 5 } & $R a_{F}$ dependence & Laboratory & Numerical & Model \\
$\frac{h_{0.95}}{L}$ & $R a_{F}{ }^{-1 / 6}$ & 2.65 & 2.87 & 3.39 \\
$\frac{U\left(h_{0.95}\right) L}{\kappa^{*}}$ & $R a_{F}{ }^{1 / 3}$ & 0.46 & 0.40 & 0.33 \\
$N u$ & $R a_{F}{ }^{1 / 6}$ & 0.82 & 0.62 & $\sim 0.75$
\end{tabular}

TABLE 3. Comparison of multiplying constants for horizontal convection, previously measured in the laboratory and evaluated in numerical solutions Mullarney et al. (2004), with those multiplying constants predicted by the theoretical recycling box model in this paper. The exponents on the Rayleigh number $R a_{F}$ from the three approaches are identical.

velocity found by Mullarney et al. (2004) for horizontal convection. This is because the empirical results and boundary-layer scaling of Mullarney et al. (2004) (and also that of Rossby 1965) are based on the vertical velocity in the thermal boundary layer, whereas (4.4) incorporates the effect of entrainment into the recycling box plume. Substituting instead the value for $w_{e}\left(\delta_{0.95}\right)$ in table 1 into (2.20) gives

$$
\frac{W_{e}\left(h_{0.95}\right) L}{\kappa^{*}}=1.83 \times 2^{1 / 6} E^{1 / 3} c^{1 / 6} \operatorname{Pr}^{1 / 6} \operatorname{Ra}_{F}{ }^{1 / 6},
$$

for the recycling box, in agreement with the previous boundary-layer scalings for horizontal convection. Applying continuity, $U h_{0.95}=W_{e}\left(h_{0.95}\right) L$, predicts the scaling for the horizontal velocity in the thermal boundary layer in the recycling box as

$$
\frac{U\left(h_{0.95}\right) L}{\kappa^{*}}=0.89 \times 2^{1 / 3} E^{2 / 3} c^{1 / 3} \operatorname{Pr}^{1 / 3} \operatorname{Ra}_{F}{ }^{1 / 3} \text {. }
$$

Substituting an appropriate value for $E$ and laboratory values for $c$ and $\operatorname{Pr}$ into (4.6) predicts that $U h_{0.95} / L=0.33 R a_{F}^{1 / 3}$, which agrees well with both the laboratory and numerical dependences for horizontal convection, $U h_{0.95} / L=0.46 R a_{F}^{1 / 3}$ and $U h_{0.95} / L=0.40 R a_{F}^{1 / 3}$, respectively, found by Mullarney et al. (2004). Following Mullarney et al. (2004), we can define for horizontal convection the Nusselt number based on the heat flux relative to conduction along the length of the box as

$$
N u=\frac{F_{T} L}{\left.\rho_{r} c_{p} \kappa^{*} \delta T\right|_{\max }},
$$


where $\left.\delta T\right|_{\max }$ is the difference between the maximum and minimum temperatures in the boundary layer. In order to extend the analogy to the recycling box model with an assumed one-dimensionality of flow outside the plume, we further assume that $\left.\delta T\right|_{\max }$ scales with the top-to-bottom temperature contrast in the recycling box, i.e. $\left.\Delta T\right|_{\max }=\left.a \delta T\right|_{\max }$. Thus, we obtain for the recycling box

$$
N u=\frac{F_{T} L a}{\left.\rho_{r} c_{p} \kappa^{*} \Delta T\right|_{\max }}=0.79 \times 2^{1 / 6} a E^{1 / 3} c^{-5 / 6} \operatorname{Pr}^{1 / 6} \operatorname{Ra}_{F}{ }^{1 / 6},
$$

and substituting an appropriate value for $E$ and laboratory values for $c$ and $\operatorname{Pr}$ into (4.8) predicts that $N u=1.00 a R a_{F}{ }^{1 / 6}$. We note that the minimum temperature obtained from our one-dimensional interior model in the recycling box, and used to calculate $\left.\Delta T\right|_{\max }$, corresponds to a horizontal average in the thermal boundary layer of the nominally two-dimensional temperature distribution used by Mullarney et al. (2004) in the calculation of $\left.\delta T\right|_{\max }$. An appropriate value of $a$ (based on the horizontal convection measurements of Mullarney et al. 2004) where the temperature in the boundary layer above the cooled region of the base was approximately uniform, but steadily increased towards the plume above the heated region of the base to enable comparison of the $N u$ prediction will lie between 0.5 and 1, but might be reasonably estimated as 0.75 (based on a linear decrease in temperature with distance from the plume above the heated region of the base). Thus, the recycling box model predicts $N u \sim 0.75 R a_{F}^{1 / 6}$, which compares well both with $N u=0.82 R a_{F}^{1 / 6}$ and $N u=0.62 R a_{F}^{1 / 6}$ found for horizontal convection by Mullarney et al. (2004) in experiments and numerical solutions, respectively, and with $N u \approx 0.35 R a^{1 / 5}$ obtained for horizontal convection by Rossby (1965) (where $R a=\left.g \alpha \delta T\right|_{\max } L^{3} / \nu \kappa^{*}$ ). The above comparisons of the recycling box model predictions with the laboratory measurements and numerical solutions of Mullarney et al. (2004) for horizontal convection are summarized in table 3.

These recycling box results are also consistent with the bounds on horizontal convection constructed by Siggers et al. (2004). Figure 3 suggests that as $\zeta_{H} \rightarrow \infty$ (i.e. $R a_{F}, R a \rightarrow \infty$, see (2.15)), the temperature variation in the interior above the boundary layer will vanish. However, as seen above, the dependence of the boundarylayer thickness and the Nusselt number upon $R a$ is weaker in the recycling box than the bounding dependences ( $R a^{-1 / 3}$ and $R a^{1 / 3}$, respectively) obtained by Siggers $e t a l$. (2004).

The dependence of the recycling box predictions upon the plume entrainment coefficient, heat input and interior diffusivity, which were extracted from (4.2)-(4.8), are summarized in table 2. We note that in (4.2)-(4.6), the solution is inviscid and therefore $\mathrm{Ra}_{F}$ and $\mathrm{Pr}$ always appear as their product. The boundary-layer analyses for horizontal convection of Rossby (1965) and Mullarney et al. (2004) are based explicitly on a buoyancy-viscous balance for the horizontal flow in the thermal boundary layer, whereas our recycling box solution is entirely inviscid, yet yields identical results (for the two-dimensional case). We suggest that this is because the flow outside the thermal boundary layer (for fluids with $\operatorname{Pr}>1$ ) is essentially inviscid and driven by entrainment into the turbulent plume (as evidenced by the much stronger Rayleigh number dependence of $\left.W_{e}(H) \sim \psi\right|_{\max }$ than of $W_{e}\left(h_{0.95}\right)$, table 2). The viscous flow in the thermal boundary layer must satisfy matching conditions (such as continuity of velocities) at the edge of the boundary layer with the external inviscid flow. However, the bulk characteristics of the thermal boundary layer, such as the driving temperature difference, the horizontal mass flux and the Nusselt number, are 
determined by the external flow. Viscosity will modify the temperature and velocity structure within the boundary layer itself, subject to these strong overall constraints. Thus, the buoyancy-viscous boundary-layer scaling analyses (Rossby 1965; Mullarney et al. 2004) cannot predict the maximum overturning streamfunction. We postulate that in high-Rayleigh-number horizontal convection, the external flow modifies the boundary-layer structure typically observed in a $\operatorname{Pr}>1$ fluid such that the thermal and momentum boundary layers are of similar thickness (as observed by Mullarney et al. 2004).

\section{Is the concept of a buoyancy-forced overturning relevant to the oceans?}

The meridional overturning circulation of the oceans carries heated surface waters poleward, where they are cooled and sink in highly localized downwellings at polar latitudes. In the downwelling plume of largest buoyancy flux (the outflow from the Weddell Sea) the cold water sinks to the bottom, spreads through much of the oceans as Antarctic Bottom Water, and is eventually returned to the surface layers by a broad slow upwelling in the interior. If the ocean is presumed to be in a steady state, the interior upwelling of cold water must be countered by downward mixing of heat (and upward mixing of density) through the full depth. The global oceans represent a very complex system that is forced by a variety of mechanisms which include buoyancy (others include wind stress and Coriolis forces influencing the mean flow, and winds and tides providing energy to turbulent mixing). However, as fundamental oceanographic questions remain concerning the importance of buoyancy-forcing in the context of the global oceans, it is instructive to consider here models for simpler overturning circulations that are buoyancy-driven.

In this section, we consider a highly idealized representation of the global oceans as a non-rotating rectangular recycling box with vertical plumes. Our aims are twofold. First, we can understand a flow in which buoyancy is clearly the only mechanism supplying momentum to the overturning circulation. Secondly, we are able to examine the robustness of our conclusions to different plume representations. We accomplish this by comparing our results with recent work treating plumes as geostrophic slope currents (Hughes \& Griffiths 2006), which is more realistic for the plumes in the oceans. Laboratory experiments (Killworth \& Turner 1982; Wong \& Griffiths 2001) suggest that the plume reaching the bottom will dominate the overall density structure, hence we consider a single plume in the recycling box model. We choose the bulk geometry and forcing for the circulation in our box to be commensurate with the global oceans. The cross-sectional area of the recycling box ocean is taken to be $3.6 \times 10^{14} \mathrm{~m}^{2}$ with an average depth of $3780 \mathrm{~m}$ (Gill 1982), and the rate of heat input is set to the poleward heat transport in the southern hemisphere oceans $\left(2 \times 10^{15} \mathrm{~W}\right.$, Houghton et al. 1996, p. 212). We take the reference density $\rho_{r}=1025 \mathrm{~kg} \mathrm{~m}^{-3}$ and the specific heat capacity $c_{p}=3990 \mathrm{~J} \mathrm{~kg}^{-1} \mathrm{~K}^{-1}$, appropriate for seawater at $20^{\circ} \mathrm{C}$ and atmospheric pressure Gill (1982), and the expansion coefficient $\alpha=2.54 \times 10^{-5} \mathrm{~K}^{-1}$ appropriate for seawater at $-2{ }^{\circ} \mathrm{C}$ (Gill 1982, this temperature being representative of the water in the Weddell Sea that feeds the sinking plumes and eventually becomes bottom water). The rate of supply of specific buoyancy to the recycling box ocean in the single ('southern hemipshere') plume is $1.2 \times 10^{5} \mathrm{~m}^{4} \mathrm{~s}^{-3}$, by (2.1).

Measurements in the ocean interior of turbulent dissipation and of vertical tracer dispersion (e.g. Gregg 1989; Ledwell et al. 1993) suggest that the rate of vertical mixing can be characterized by a diffusivity of order $10^{-5} \mathrm{~m}^{2} \mathrm{~s}^{-1}$. (The density structure in the oceans has been previously described by a simple one-dimensional 


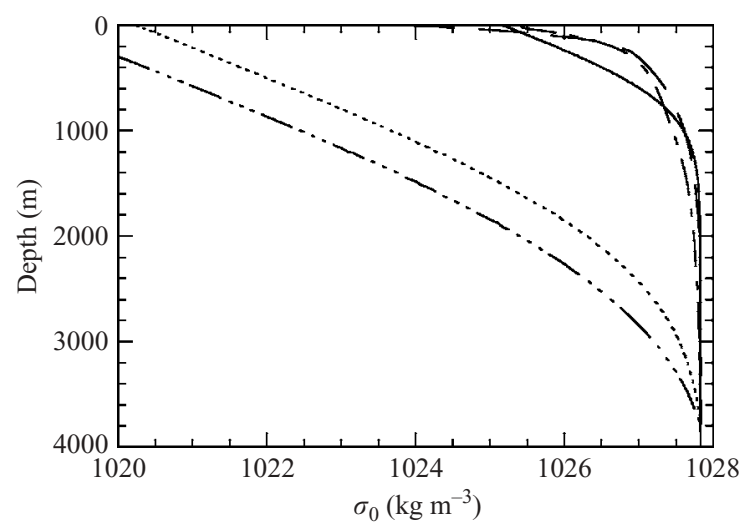

FIGURE 6. Density profiles predicted by the recycling box model (for $\kappa^{*}=10^{-5} \mathrm{~m}^{2} \mathrm{~s}^{-1}$, and the geometric parameters and seawater properties discussed in the text) for a single axisymmetric 'half-plume' $(-\cdots-)$, a single axisymmetric isolated plume $(\cdots)$ and the geostrophic slope plume of Hughes \& Griffiths (2006) (-). Potential density $\left(\sigma_{0}\right)$ profiles averaged from the Levitus 1994 dataset (http://iridl.ldeo.columbia.edu/SOURCES/.LEVITUS94/) for the northern (-) and southern (- - - ) hemisphere oceans are shown for comparison. The predicted density profiles are referenced to the global average potential density measured below depths of $3000 \mathrm{~m}$ $\left(\approx 1027.83 \mathrm{~kg} \mathrm{~m}^{-3}\right)$.

balance of advection and diffusion (cf. (2.8)), from which the measured abyssal gradients imply a diffusivity of order $10^{-4} \mathrm{~m}^{2} \mathrm{~s}^{-1}$ (Munk 1966; Munk \& Wunsch 1998). However, as no account was taken in these balances of entrainment into the plumes, we use the measured $\kappa^{*}=10^{-5} \mathrm{~m}^{2} \mathrm{~s}^{-1}$ here.) By (A 12), we calculate $\zeta_{H} \approx 2.5$ and $\zeta_{H} \approx 3.1$ for a single axisymmetric 'half-plume' and a single axisymmetric isolated plume, respectively, in this idealized recycling box ocean. Although we expect our axisymmetric plume solution to be only approximate at these values of $\zeta_{H}$, the predicted density structure is compared in figure 6 with the measured (horizontally averaged) density profile in the oceans and the solution for the same governing parameters of the more sophisticated geostrophic slope plume model of Hughes \& Griffiths (2006). In addition, the axisymmetric half-plume and isolated plume solutions predict overturning rates of $4.8 \times 10^{6}$ and $7.3 \times 10^{6} \mathrm{~m}^{3} \mathrm{~s}^{-1}$, respectively, which compares with $18.4 \times 10^{6} \mathrm{~m}^{3} \mathrm{~s}^{-1}$ predicted by Hughes \& Griffiths (2006) and the range of total bottom water formation rates $7-30 \times 10^{6} \mathrm{~m}^{3} \mathrm{~s}^{-1}$ estimated from observational studies. The effect that plume geometry has on the amount of entrainment is apparent upon comparison of the various predictions. At any given level, the axisymmetric 'halfplume' has less circumferential area than the axisymmetric isolated plume across which to entrain ambient water. Thus, the total volume of ambient water entrained (which is equal to the maximum overturning flux) is smaller and the top-to-bottom density difference is larger. The model of Hughes \& Griffiths (2006), which allows for the plume to expand laterally on a topographic slope owing to baroclinic instability, has a much greater surface area at shallow to intermediate depths than an axisymmetric plume. Thus, in their model, the density increase with depth in the interior occurs more rapidly and the maximum overturning flux is greater. The boundary-layer thicknesses $h_{e}$ estimated from the density profiles in figure $6(1700$ and $1470 \mathrm{~m}$ for the axisymmetric 'half-plume' and the axisymmetric isolated plume, respectively, and $550 \mathrm{~m}$ for the geostrophic slope plume) are consistent with the above entrainment characteristics. It is, however, worthy of note that none of these boundary-layer 
thicknesses in the recycling box ocean follow the scaling for horizontal convection of Rossby (1965) and Mullarney et al. (2004), which yields a much smaller thickness of $O(100) \mathrm{m}$. These previous boundary-layer scalings are based on the volume flux in a two-dimensional thermal boundary layer in horizontal convection. However, the sinking regions in the oceans more closely resemble localized three-dimensional plumes, which therefore cannot accommodate the volume flux from the near surface that is required by the boundary-layer scalings based on a two-dimensional flow. Accordingly, entrainment into the localized sinking plumes takes place over a greater range of depths, thus increasing the boundary-layer thickness as figure 6 shows.

None of the recycling box models take account of mechanisms of vertical buoyancy transport other than by entrainment into plumes and (uniform) diffusion. In the ocean, however, additional mechanisms such as wind-driven upwelling and locally enhanced mixing (owing to processes such as breaking internal waves and the interaction of internal tides with topography) are present and likely to account for some of the discrepancy in figure 6 between measurements and the predictions based on simple entraining plume models. Nevertheless, it is apparent that entrainment into localized plumes is likely to be very significant for the global oceans and that accurate modelling of plume geometry is an important step in understanding the density structure of the oceans.

\section{Conclusions}

We have developed a simple model to describe high-Rayleigh-number horizontal convection by taking advantage of the features that this flow has in common with a special class of 'filling box' flows. Specifically, differential heating along one horizontal boundary in horizontal convection gives rise to a localized vertical turbulent plume that ventilates the box volume. As in the filling box, entrainment into this plume maintains a stable density stratification throughout the box depth (we note, however, that compared with a geometrically identical filling box forced with the same buoyancy flux, the stratification beyond the thermal boundary layer is much weaker in the case of horizontal convection). With zero net heat input into the box in horizontal convection, the density profile is steady and maintained by diffusion against the vertical motion that ventilates the box volume. In our model, we have considered both two-dimensional and axisymmetric localized plumes that are assumed to have a point source at the differentially heated surface, and we represent the box interior as horizontally uniform. Although the circulation in horizontal convection is driven by horizontal density gradients along the differentially heated boundary, comparison with measurements shows that our model (in which these gradients are condensed to the edge of the plume) correctly predicts many aspects of the flow, including the vertical density structure, the thermal boundary-layer thickness adjacent to the heated boundary, the magnitude of the circulation, and the dependence of Nusselt number upon Rayleigh number.

Our model provides a basis for investigating the role that horizontal convection may play in the context of the meridional overturning circulation in the global oceans. Although additional effects of importance to the ocean circulation, such as wind-forcing, have obviously been neglected, our results support the assertion that horizontal convection is likely to be of importance in the overturning circulation, in contrast to some popular views in the oceanographic literature. The models predict global overturning rates consistent with that measured. A particularly notable result is that entrainment into localized plumes is expected to affect the global vertical 
density structure in the oceans. Although the vertical plumes considered in this paper are shown to be an oversimplified representation of the sinking regions in the oceans, the sensitivity of our results to plume geometry serves to illustrate the importance of understanding such sinking regions.

We thank three anonymous reviewers for their comments. The work was finded in part by the Australian Research Council (DP0664115).

\section{Appendix. Axisymmetric plume}

We outline here our model for the recycling box driven by a turbulent axisymmetric plume whose radius is assumed to be much smaller than the lateral dimensions of the box. The cross-sectional area of the box and the specific buoyancy flux in the plume is defined to be $2 A(z)$ and $2 F_{0}=F_{h}$, respectively. Thus, an axisymmetric 'half-plume' against a vertical boundary can be represented with a specific buoyancy flux of $F_{0}$ in a box of area $A(z)$. If the mean vertical velocity and density anomaly through the plume cross-section take the Gaussian form in (2.2) and (2.3), then the equations describing conservation of volume, momentum and buoyancy in the plume and conservation of volume in the box interior are (Baines \& Turner 1969)

$$
\begin{gathered}
\frac{\mathrm{d}}{\mathrm{d} z}\left[R^{2} W_{p}\right]=2 R U_{e}=2 E R W_{p}, \\
\frac{\mathrm{d}}{\mathrm{d} z}\left[\frac{R^{2} W_{p}^{2}}{2}\right]=R^{2} \frac{\boldsymbol{g} \cdot \hat{z}\left(\rho_{p}-\rho_{e}\right)}{\rho_{r}}, \\
\frac{\mathrm{d}}{\mathrm{d} z}\left[\frac{R^{2} W_{p} \boldsymbol{g} \cdot \hat{z}\left(\rho_{p}-\rho_{e}\right)}{2 \rho_{r}}\right]=R^{2} W_{p} \frac{\mathrm{d}}{\mathrm{d} z}\left[\frac{\boldsymbol{g} \cdot \hat{z}\left(\rho_{r}-\rho_{e}\right)}{\rho_{r}}\right], \\
\frac{\pi R^{2} W_{p}}{2}=-A(z) W_{e},
\end{gathered}
$$

respectively. The vertical advection-diffusion balance in the box interior is given by

$$
A(z) W_{e} \frac{\mathrm{d} \rho_{e}}{\mathrm{~d} z}=\frac{\mathrm{d}}{\mathrm{d} z}\left[A(z) \kappa^{*}(z) \frac{\mathrm{d} \rho_{e}}{\mathrm{~d} z}\right] .
$$

The plume is approximated as a point source of buoyancy with no volume flux at the forcing boundary so that the boundary conditions are

$$
\begin{gathered}
{\left.\left[R^{2} W_{p}\right]\right|_{z=0}=0,} \\
R(0)=0 .
\end{gathered}
$$

Therefore,

$$
W_{e}(0)=0
$$

by (A 4). Further, we assume the horizontal velocity $U_{e}$ at $z=0$ to be zero (i.e. a no-slip boundary) and, by (A 1), entrainment into the plume at $z=0$ is also zero,

$$
\left.\left[R W_{p}\right]\right|_{z=0}=0 .
$$

As described in $\S 2.3$, a stabilizing buoyancy flux is supplied to the box either at a rate $F_{c}=F_{0}=F_{h}$ for a half-plume or at a rate $F_{c}=2 F_{0}=F_{h}$ for an isolated plume. We choose to distribute the stabilizing flux uniformly over the forcing boundary at $z=0$. 
Upon integrating the specific buoyancy flux per unit area over the plume cross-section and taking the limit as $z \rightarrow 0$, the boundary condition may be written

$$
F_{0}=\left.\frac{\pi}{4}\left[\frac{R^{2} W_{p} \boldsymbol{g} \cdot \hat{z}\left(\rho_{p}-\rho_{e}\right)}{\rho_{r}}\right]\right|_{z=0}=\frac{\boldsymbol{g} \cdot \hat{z} A(0) \kappa^{*}}{\rho_{r}} \frac{d \rho_{e}(0)}{d z} .
$$

Equations (A 1)-(A 5) are strictly valid only for the part of the box volume that excludes the plume outflow, which is neglected here. As discussed in $\S 2.3$, we determine the validity of our approximate solution by checking whether boundary conditions at $z=H$ of zero specific buoyancy flux hold in each of the plume and the interior, i.e.

$$
F(H)=\left.\frac{\pi}{4}\left[\frac{R^{2} W_{p} \boldsymbol{g} \cdot \hat{z}\left(\rho_{p}-\rho_{e}\right)}{\rho_{r}}\right]\right|_{z=H}=\frac{\boldsymbol{g} \cdot \hat{z} A(H) \kappa^{*}}{\rho_{r}} \frac{\mathrm{d} \rho_{e}(H)}{\mathrm{d} z}=0 .
$$

As in $\S 2.4$, we define the dimensionless variables

$$
\begin{aligned}
z & =2^{-1 / 2} \pi^{-1 / 4} E^{-1 / 2} F_{0}^{-1 / 8}\left(\kappa^{*} A\right)^{3 / 8} \zeta, \\
R & =2^{1 / 2} \pi^{-1 / 4} E^{1 / 2} F_{0}^{-1 / 8}\left(\kappa^{*} A\right)^{3 / 8} r, \\
\frac{g \cdot \hat{z}\left(\rho_{p}-\rho_{e}\right)}{\rho_{r}} & =2^{1 / 2} \pi^{-1 / 4} E^{-1 / 2} F_{0}{ }^{7 / 8}\left(\kappa^{*} A\right)^{-5 / 8} f_{p}, \\
\frac{\boldsymbol{g} \cdot \hat{z}\left(\rho_{r}-\rho_{e}\right)}{\rho_{r}} & =2^{-1 / 2} \pi^{-1 / 4} E^{-1 / 2} F_{0}{ }^{7 / 8}\left(\kappa^{*} A\right)^{-5 / 8} f_{e}, \\
W_{p} & =2^{1 / 2} \pi^{-1 / 4} E^{-1 / 2} F_{0}^{3 / 8}\left(\kappa^{*} A\right)^{-1 / 8} w_{p}, \\
W_{e} & =2^{1 / 2} \pi^{1 / 4} E^{1 / 2} F_{0}{ }^{1 / 8} \kappa^{* 5 / 8} A^{-3 / 8} w_{e},
\end{aligned}
$$

so that (A 1)-(A 5) become

$$
\begin{gathered}
\frac{\mathrm{d}}{\mathrm{d} \zeta}\left(r^{2} w_{p}\right)=r w_{p}, \\
\frac{\mathrm{d}}{\mathrm{d} \zeta}\left(r^{2} w_{p}^{2}\right)=r^{2} f_{p}, \\
\frac{\mathrm{d}}{\mathrm{d} \zeta}\left(r^{2} w_{p} f_{p}\right)=r^{2} w_{p} \frac{\mathrm{d} f_{e}}{\mathrm{~d} \zeta}, \\
r^{2} w_{p}=-\frac{A(\zeta)}{A(0)} w_{e}, \\
\frac{A(\zeta)}{A(0)} w_{e} \frac{\mathrm{d} f_{e}}{\mathrm{~d} \zeta}=\frac{\mathrm{d}}{\mathrm{d} \zeta}\left[\gamma(\zeta) \frac{\mathrm{d} f_{e}}{\mathrm{~d} \zeta}\right],
\end{gathered}
$$

where

$$
\gamma=\frac{\left.\kappa^{*} A\right|_{\zeta}}{\left.\kappa^{*} A\right|_{\zeta=0}}
$$

Following the method in $\S 2.4$, we obtain an ordinary differential equation for the dimensionless specific buoyancy flux in the plume $\phi(\zeta)=r^{2} w_{p} f_{p}$,

$$
\frac{\mathrm{d}}{\mathrm{d} \zeta}\left[\frac{1}{\phi} \frac{\mathrm{d} \phi}{\mathrm{d} \zeta}\right]+2^{1 / 4}(1-\phi)^{1 / 4}=0
$$


which becomes singular at $\zeta=1$. We integrate (A 24) from $\zeta^{\prime}=0$ to $\zeta^{\prime}=\zeta$ to obtain a first-order o.d.e. for $\phi$,

$$
\frac{\mathrm{d} \phi}{\mathrm{d} \zeta}= \pm 2^{9 / 8} \phi\left\{\tanh ^{-1}\left[(1-\phi)^{1 / 4}\right]+\tan ^{-1}\left[(1-\phi)^{1 / 4}\right]-2(1-\phi)^{1 / 4}\right\}^{1 / 2} .
$$

As $\phi$ decreases with $\zeta$, we take the negative root of (A 25) and integrate numerically subject to the boundary condition $\phi=1$ at $\zeta=0$.

Solutions for the dimensionless density anomaly and vertical velocity in the interior are then given by (2.35) and (2.36), while the dimensionless vertical velocity and density anomaly in the plume are given by (2.37) and (2.38), and the dimensionless plume radius by

$$
r=-\frac{\mathrm{d} \phi / \mathrm{d} \zeta}{2^{1 / 4} \phi(1-\phi)^{1 / 4}}
$$

\section{REFERENCES}

BAINES, W. D. 1985 Entrainment by a buoyant jet flowing along vertical walls. J. Hydraul. Res. 23, 221-228.

BAINES, W. D. \& TuRnER, J. S. 1969 Turbulent buoyant convection from a source in a confined region. J. Fluid Mech. 37, 51-80.

Beardsley, R. C. \& Festa, J. F. 1972 A numerical model of convection driven by a surface stress and non-uniform heating. J. Phys. Oceanogr. 2, 444-455.

Gill, A. E. 1982 Atmosphere-Ocean Dynamics. Academic.

GregG, M. C. 1989 Scaling turbulent dissipation in the thermocline. J. Geophys. Res. 94, 9686-9698.

Hignett, P., Ibbetson, A. \& Killworth, P. D. 1981 On rotating thermal convection driven by non-uniform heating from below. J. Fluid Mech. 109, 161-187.

Houghton, J. T., Meira Filho, L. G., Callander, B. A., Harris, N., Kattenberg, A. \& Maskell, K. 1996 Climate Change 1995: The Science of Climate Change. Cambridge University Press.

Hughes, G. O. \& Griffiths, R. W. 2006 A simple convective model of the global overturning circulation, including effects of entrainment into sinking regions. Ocean Modell. 12, 46-79.

JefFreYs, H. T. 1925 On fluid motions produced by differences of temperature and humidity. Q. $J$. R. Met. Soc. 51, 347-356.

Killworth, P. D. \& Manins, P. C. 1980 A model of confined thermal convection driven by non-uniform heating from below. J. Fluid Mech. 98, 587-607.

Killworth, P. D. \& Turner, J. S. 1982 Plumes with time-varying buoyancy in a confined region. Geophys. Astrophys. Fluid Dyn. 20, 265-291.

Ledwell, J. R., Watson, A. J. \& LaW, C. S. 1993 Evidence for slow mixing across the pycnocline from an open-ocean tracer release experiment. Nature 364, 701-703.

Linden, P. F. 1999 The fluid mechanics of natural ventilation. Annu. Rev. Fluid Mech. 31, 201-238.

Manins, P. C. 1973 A filling box model of the deep circulation of the Red Sea. Mém. Soc. R. Sci. Liége 6, 153-166.

Manins, P. C. 1979 Turbulent buoyant convection from a source in a confined region. J. Fluid Mech. 91, 765-781.

Mullarney, J. C., Griffiths, R. W. \& Hughes, G. O. 2004 Convection driven by differential heating at a horizontal boundary. J. Fluid Mech. 516, 181-209.

Munk, W. H. 1966 Abyssal recipes. Deep-Sea Res. 13, 707-730.

Munk, W. H. \& Wunsch, C. 1998 Abyssal recipes II: energetics of tidal and wind mixing. Deep-Sea Res. I 45, 1977-2010.

Paparella, F. \& Young, W. R. 2002 Horizontal convection is non-turbulent. J. Fluid Mech. 466, 205-214.

Peterson, W. H. 1979 A steady thermohaline convection model. PhD thesis, University of Miami.

PierCe, D. W. \& Rhines, P. B. 1996 Convective building of a pycnocline: laboratory experiments. J. Phys. Oceanogr. 26, 176-190. 
Rossby, H. T. 1965 On thermal convection driven by non-uniform heating from below: an experimental study. Deep-Sea Res. 12, 9-16.

Rossby, H. T. 1998 Numerical experiments with a fluid non-uniformly heated from below. Tellus 50, 242-257.

Siggers, J. H., Kerswell, R. R. \& Balmforth, N. J. 2004 Bounds on horizontal convection. J. Fluid Mech. 517, 55-70.

Stommel, H. 1962 On the smallness of sinking regions in the ocean. Proc. Nat. Acad. Sci. Washington 48, 766-772.

Turner, J. S. 1973 Buoyancy Effects in Fluids. Cambridge University Press.

TURNER, J. S. 1980 Differentiation and layering in magma chambers. Nature 285, 213-215.

WANG, W. \& HuANG, R. X. 2005 An experimental study on thermal convection driven by horizontal differential heating. J. Fluid Mech. 540, 49-73.

Wong, A. B. D. \& Griffiths, R. W. 2001 Stratification and convection produced by multiple plumes. Dyn. Atmos. Oceans 30, 101-123. 